\title{
Revised Baade-Wesselink Analysis of RR Lyrae Stars
}

\author{
Carla Cacciari ${ }^{1}$, Gisella Clementini ${ }^{1}$, Fiorella Castelli ${ }^{2}$, Fabrizio \\ Melandri ${ }^{3}$
}

\begin{abstract}
We have applied the Baade-Wesselink method to two field RR Lyrae stars, i.e. SW And and RR Cet, and derived their distances and physical parameters. With respect to previous $\mathrm{B}-\mathrm{W}$ analyses we have applied the following improvements: i) use of all sets of available data, after proper comparison for homogeneity and compatibility; ii) use of the most recent and accurate model atmospheres, with turbulent velocity $V_{\text {turb }}=4 \mathrm{~km} \mathrm{~s}^{-1}$ and the no-overshooting approximation, and comparison with other treatments of convection; iii) use of the instantaneous gravity along the pulsation cycle rather than the mean value; iv) comparison with modified radial velocity curves according to various assumptions on radial velocity gradients in the atmosphere; and v) careful reanalysis of the temperature scale. The main aim of this study is to evaluate the effect of the above items on the B-W results and verify whether any (or a combination) of them can possibly account for the discrepancy of the absolute magnitude zero-point with respect to other independent determinations.
\end{abstract}

\section{Introduction}

The distance scale problem is not solved yet, the dichotomy between long and short distance scales becoming even more clear-cut. In favour of the short distance scale (i.e. faint $\left.M_{V}(\mathrm{RR}),(m-M)_{\mathrm{LMC}} \leq 18.3\right)$ are the studies on RR Lyrae statistical parallaxes (Gould \& Popowski 1998), local RR Lyrae kinematics (Martin \& Morrison 1998), eclipsing binaries in the LMC (Udalski et al. 1998, but see also Guinan et al. 1998 for a contrasting result), red clump stars (Cole 1998), and Hipparcos parallaxes of field HB stars (Gratton 1998). On the other hand, in favour of the long distance scale (i.e. bright $M_{V}(\mathrm{RR})$, $\left.(m-M)_{\text {LMC }} \geq 18.5\right)$ are the studies on sd-MS fitting in globular clusters (Gratton et al. 1997; Reid 1997; Chaboyer et al. 1998; Pont et al. 1998; Grundahl, VandenBerg, \& Andersen 1998; Carretta et al. 1999), RGB bump stars in globular clusters (Ferraro et al. 1999), tip of the RGB (Lee, Freedman, \& Madore 1993), RR Lyrae period-shift effect (Sandage 1993), RR Lyrae double-mode pulsators (Kovács \& Walker 1998), Hipparcos parallaxes of Cepheids (Feast \& Catchpole 1997), and SN1987A in the LMC (Panagia 1998).

\footnotetext{
${ }^{1}$ Osservatorio Astronomico, Via Ranzani 1, I-40127 Bologna, Italy

${ }^{2}$ Osservatorio Astronomico, Via Tiepolo 11, I-34131 Trieste, Italy

${ }^{3}$ Dipartimento di Astronomia, Via Ranzani 1, I-40127 Bologna, Italy
} 
The Baade-Wesselink (B-W) analysis of RR Lyrae stars can help solve this problem. For a review and general description of this method and its assumptions and approximations see, for example, Gautschy (1987).

Previous B-W analyses were performed on 29 field RR Lyrae stars by a few independent groups, e.g. Liu \& Janes (1990), Jones et al. (1992), Cacciari, Clementini, \& Fernley (1992), and Skillen et al. (1993). These references are only indicative and are by no means exhaustive or complete. The results of these and several more B-W studies have been reanalysed and summarised by Fernley (1994) and Fernley et al. (1998) who find a relation $M_{V}(\mathrm{RR})=$ $0.20 \pm 0.04[\mathrm{Fe} / \mathrm{H}]+0.98 \pm 0.05$ between the absolute visual magnitude and the metallicity of the RR Lyrae stars. This result is intermediate, but closer to the short distance scale, corresponding to $(m-M)_{\text {LMC }} \sim 18.34$.

The previous B-W analyses were based on: i) Kurucz (1979) model atmospheres, with turbulent velocity $V_{\text {turb }}=2 \mathrm{~km} \mathrm{~s}^{-1}$; ii) semi-empirical temperature calibration; iii) mostly infrared (i.e. $K$ ), but also visual $V, R$ and $I$ photometry; iv) the use of an average value for gravity (usually $\log g=2.75$ ); v) a restricted phase range for fitting (usually 0.35 to 0.80 ); vi) the barycentric (also called $\gamma$-) velocity derived as simple integration over the entire pulsation cycle; and vii) a constant projection factor (1.38) to transform radial into pulsational velocities.

Several model atmospheres are now available, with: improved opacities; different treatments of convection; different values of turbulent velocity; more accurate temperature and $B C$ calibrations. The aim of the present work is to test what the effect is of these new models and calibrations, as well as of other assumptions, on the B-W results for RR Lyrae stars.

\section{Our Re-analysis: Assumptions and Approximations}

\subsection{New Elements}

- Model atmospheres. We have used the following sets of model atmospheres: i) Kurucz (1995) with MLT+overshooting treatment of convection, $V_{\text {turb }}=2$ and $4 \mathrm{~km} \mathrm{~s}^{-1},[\mathrm{~m} / \mathrm{H}]=0.0$; ii) Castelli $(1999 \mathrm{~b})$ with MLT without overshooting treatment of convection, $[\mathrm{m} / \mathrm{H}]=0.0$ and $V_{\text {turb }}=2 \mathrm{~km} \mathrm{~s}^{-1}$, and $[\mathrm{m} / \mathrm{H}]=-1.5$ and $V_{\text {turb }}=2$ and $4 \mathrm{~km} \mathrm{~s}^{-1}$. The models at $[\mathrm{m} / \mathrm{H}]=-1.5$ are enhanced in $\alpha$-elements by $\left[\alpha / \alpha_{\odot}\right]=+0.4$, iii) Castelli (1999a) experimental models with no convection. These models do not have physical meaning, but are only intended to mimic the effects of recent treatments of convection, e.g. MLT with $1 / \mathrm{H}=0.5$ instead of 1.25 (Fuhrmann, Axer, \& Gehren 1993), or the Canuto \& Mazzitelli (1992) approximation that predicts a very low convection or zero convection for stars with $T_{\text {eff }} \geq 7000 \mathrm{~K}$, and that has been suggested to provide a better match to the data (see Gardiner, Kupka, \& Smalley 1999 for a recent re-discussion of this issue). The no-convection models are available for $[\mathrm{m} / \mathrm{H}]=0.0$ and -1.5 and $V_{\text {turb }}$ $=2 \mathrm{~km} \mathrm{~s}^{-1}$.

- Gravities. The values of $\log g$ have been calculated at each phase-step from the radius percentage variation (assuming $\Delta \mathrm{R} /\langle R\rangle \sim 15 \%$ ) plus the acceleration component derived from the radial velocity curve. The zero-point was set from theoretical ZAHB models, i.e. $\log g=2.86$ at the phase corresponding to average radius. Note that all ZAHB models give average $\log g=2.86 \pm 0.01$ (Dorman, Rood, \& O'Connell 1993; Sweigart 1997 with no He-mixing; Chieffi, Limongi, 
\& Straniero 1998); with the only exception being Sweigart (1997) models with He-mix $=0.10$ which give $\log g=2.75$, but may not be applicable to our field stars. - Semi-empirical $\mathbf{T}_{\text {eff }}$ and BC calibration. We have used the Montegriffo et al. (1998) semi-empirical $B C_{V}$ and $B C_{K}$ calibration and temperature scale for Pop II giants that are based on RGB and HB stars in 10 globular clusters.

- Gamma-velocity. The default value of the $\gamma$-velocity was estimated from integration of the observed radial velocity curve over the entire pulsation cycle, as it was done in all previous B-W studies. However, Oke, Giver, \& Searle (1962) had suggested for SU Dra that velocity gradients may exist in the atmosphere, and proposed to take them into account by correcting the observed radial velocities of a positive quantity that would vary about linearly between phase 0.95 and 0.40 . On the other hand, Jones et al. (1987) found no observational evidence of velocity gradients among weak metal lines in X Ari, at least within their observational errors $\left( \pm 2 \mathrm{~km} \mathrm{~s}^{-1}\right)$. Chadid \& Gillet (1998), however, did seem to find some evidence of differential velocities among weak metal lines in RR Lyr: the radial velocity curve from the Fe II $(\lambda 4923.9)$ line shows a slightly larger amplitude than that from the $\mathrm{Fe} \mathrm{I}(\lambda 4920.5)$ line which forms a little deeper in the atmosphere. A similar effect was found between $\mathrm{Ba}$ II and $\mathrm{Ti}$ II lines, and is related to the presence of strong shocks. Therefore, in addition to the default $\gamma$-velocity calculation from the observed RV curve, we have simulated two other cases, $\gamma-1$ where the RV curve has been corrected as suggested by Oke et al. (1962), albeit by a much smaller amount $\left(+2.0 \mathrm{~km} \mathrm{~s}^{-1}\right.$ at most), and $\gamma-2$ where the amplitude of the RV curve has been stretched by $\pm 5 \mathrm{~km} \mathrm{~s}^{-1}$ at the phases of maximum and minimum RV. These simulations are only numerical experiments and do not intend to provide realistic answers to the problem of radial velocity gradients in the atmosphere: the radial velocities for the RR Lyrae stars are derived from a large number of weak metal lines, and it is still totally unclear which correction (if any) should be applied to the average values in the presence of velocity gradients among some of these lines.

\subsection{Old Assumptions}

- The $p$-factor. The factor used to transform radial into pulsational velocity has been assumed to be 1.38, as in Fernley (1994). It might be a few percent smaller; this conservative assumption gives the brightest possible luminosity as a function of $p$.

- Fitting phase interval. As with all previous applications of the B-W method, the fitting has not been performed on the entire pulsation cycle, but on a restricted phase interval appropriate for each star: $0.30-0.80$ for SW And, for best stability of results; and $0.25-0.70$ for RR Cet, to avoid shock-perturbed phases.

\section{Results on SW And and RR Cet, and Discussion}

- SW And. We have used all possible and compatible data from the literature, i.e. $B V R I K$ and $u v b y$ photometry, and RVs. The adopted input parameters for this star are $[\mathrm{Fe} / \mathrm{H}]=0.0, \mathrm{E}(B-V)=0.09, \gamma$-velocity $=-19.94 \mathrm{~km} \mathrm{~s}^{-1}$ and $\left\langle V_{0}\right\rangle=9.44$. 
The default model calibration on Vega led to systematically hotter temperatures (by $\sim 180 \mathrm{~K})$ from the $(B-V)$ colours with respect to all other colours. This difference has no physical justification, and is only due to a behaviour of the models that was already known and commented on by Castelli (1999b). It is worth mentioning that this hotter temperature by $\sim 180 \mathrm{~K}$ leads to a brighter $M_{V}$ by $\sim 0.12 \mathrm{mag}$ using the combination $V$ and $B-V$ in the B-W analysis, and by only $\sim 0.02 \mathrm{mag}$ using $K$ and $B-V$. It is important to note that the use of the $I$ magnitude with any colour, in particular $V-K$, is the least affected by temperature uncertainties, and provides the most stable results.

In Table 1 we summarise the values of $M_{V}$ that result from the use of the $K$ magnitude and the various colours and models. The models are labelled as: K2 and $\mathrm{K} 4$ for Kurucz (1995) with $V_{\text {turb }}=2$ and $4 \mathrm{~km} \mathrm{~s}^{-1}$ respectively; K2-nover and K2-noconv for Castelli $(1999 \mathrm{a}, \mathrm{b}) V_{\text {turb }}=2 \mathrm{~km} \mathrm{~s}^{-1}$ and the no-overshooting and no-convection approximations respectively; Montegriffo for the Montegriffo et al. (1998) temperature scale and $B C_{K}$ calibration.

Table 1. Determinations of $M_{V}$ for SW And

\begin{tabular}{lcccccc}
\hline Model & $b-y$ & $B-V$ & $V-R$ & $V-I$ & $V-K$ & $R-K$ \\
\hline K2 & 1.11 & 0.83 & 1.02 & 1.07 & $\mathbf{0 . 9 3}$ & 0.97 \\
K4 & 1.09 & 0.91 & 1.03 & 1.05 & $\mathbf{0 . 9 2}$ & 0.95 \\
K2-nover & 1.08 & 0.84 & 0.93 & 0.99 & $\mathbf{0 . 9 6}$ & 0.97 \\
K2-noconv & 0.62 & 0.40 & 0.46 & 0.51 & $\mathbf{0 . 4 8}$ & 0.49 \\
Montegriffo & & & & & $\mathbf{0 . 9 4}$ & \\
\hline
\end{tabular}

Table 2. Determinations of $M_{V}$ for RR Cet

\begin{tabular}{lccccc}
\hline Model & $B-V$ & $V-R$ & $V-I$ & $V-K$ & $R-K$ \\
\hline K2-nover & 0.54 & 0.44 & 0.47 & $\mathbf{0 . 5 7}$ & 0.60 \\
K2-noconv & 0.53 & 0.42 & 0.45 & $\mathbf{0 . 5 5}$ & 0.59 \\
K4-nover & 0.51 & 0.41 & 0.45 & $\mathbf{0 . 5 5}$ & 0.59 \\
K2/K4-nover & 0.64 & 0.48 & 0.51 & $\mathbf{0 . 5 8}$ & 0.60 \\
K4-nover $\gamma$-1 & 0.54 & 0.44 & 0.48 & $\mathbf{0 . 5 8}$ & 0.61 \\
K4-nover $\gamma$-2 & 0.35 & 0.25 & 0.30 & $\mathbf{0 . 3 9}$ & 0.43 \\
Montegriffo & 0.48 & & & $\mathbf{0 . 5 6}$ & \\
\hline
\end{tabular}

We note that: i) The use of the $b-y$ colours yields unreliable results because the angular diameter curve is distorted and the fitting to the linear radius curve is poor. ii) The no-convection models yield much brighter magnitudes, the exact amount of "brightening" depending on the details of the convection treatment. iii) All models, except the no-convection ones, yield consistent results with the empirical relation by Montegriffo et al. (1998). iv) The present results, except the no-convection ones, are consistent with the previous determination of $M_{V}$ for SW And, i.e. 0.94 (Fernley 1994).

- RR Cet. We have used all compatible BVRIK photometry and radial velocities from the literature. The adopted input parameters for this star are 
$[\mathrm{Fe} / \mathrm{H}]=-1.5, \mathrm{E}(B-V)=0.05, \gamma$-velocity $=-74.46 \mathrm{~km} \mathrm{~s}^{-1}$ and $\left\langle V_{0}\right\rangle=9.59$. The default model calibration on Vega for the $[\mathrm{Fe} / \mathrm{H}]=-1.5$ models led to consistent temperatures within $\sim 100 \mathrm{~K}$ from all colours. In Table 2 we summarise the values of $\mathrm{M}_{V}$ for $\mathrm{RR}$ Cet that result from the use of the $K$ magnitude and the various colours and models. In addition to the models described also in Table 1, we have here a few other cases: K4-nover for Castelli (1999b) $V_{\text {turb }}=4 \mathrm{~km}$ $\mathrm{s}^{-1}$ and the no-overshooting approximation; K2/K4-nover for $V_{\text {turb }}=4 \mathrm{~km} \mathrm{~s}^{-1}$ only at phases $0.60-1.00$ and $V_{\text {turb }}=2 \mathrm{~km} \mathrm{~s}^{-1}$ elsewhere; $\gamma-1$ for a corrected RV curve by $+2 \mathrm{~km} \mathrm{~s}^{-1}$ at the phase of minimum RV; $\gamma-2$ for a corrected RV curve whose amplitude has been stretched by $\pm 5 \mathrm{~km} \mathrm{~s}^{-1}$.

We note that: i) The no-convection models seem to have no significant effect on the derived magnitudes. ii) The use of $V_{\text {turb }}=4 \mathrm{~km} \mathrm{~s}^{-1}$ all over the fitting phase interval yields insignificantly brighter magnitudes (by $\leq 0.03 \mathrm{mag}$ ) with respect to the case at $V_{\text {turb }}=2 \mathrm{~km} \mathrm{~s}^{-1}$. On the other hand, setting $V_{\text {turb }}$ $=4 \mathrm{~km} \mathrm{~s}^{-1}$ only at phases $0.60-1.00$ and $V_{\text {turb }}=2 \mathrm{~km} \mathrm{~s}^{-1}$ elsewhere leads to somewhat fainter magnitudes, the effect being stronger in the bluer colours. iii) Correcting the RV curve as suggested by Oke et al. (1962) by at most $+2 \mathrm{~km} \mathrm{~s}^{-1}$ at the phase of minimum RV (case $\gamma-1$ ) leads to slightly fainter magnitudes. iv) Correcting the RV curve by stretching the amplitude in analogy to the behaviour of Fe II and Ba II lines (case $\gamma-2$ ) leads to brighter magnitudes. The amount of brightening we have estimated is however too large, since the correction we have applied $\left( \pm 5 \mathrm{~km} \mathrm{~s}^{-1}\right)$ was exaggerated for the sake of computation. v) All models yield consistent results with the empirical relation by Montegriffo et al. (1998). vi) The present results are slightly brighter than the previous determination of $M_{V}$ for RR Cet, i.e. 0.68 (Fernley 1994).

\section{Conclusions}

The meaning of our simulations with the no-convection models is only to point out that, if the effect of convection were indeed overestimated by the present MLT, a more correct treatment with a reduced impact of convection would lead to brighter magnitudes for solar-metallicity stars, but would have lesser consequences on metal-poor stars. However, Gardiner et al. (1999) reach no definitive conclusion on this subject, and suggest that the classical treatment of convection with $1 / \mathrm{H}=1.25$ may still be the best approach in the temperature range $6000-7000 \mathrm{~K}$ which is relevant for $\mathrm{RR}$ Lyrae stars around minimum light.

The corrections to the radial velocity curves simulated with RR Cet are quite arbitrary and not sufficiently supported by observational evidence. They are only intended as numerical experiments to test their effect on the derived magnitudes.

The present results are quite compatible with the results from previous analyses, and do not support a significantly brighter zero-point for the RR Lyrae luminosity scale.

\section{References}

Cacciari, C., Clementini, G., \& Fernley, J. 1992, ApJ, 396, 219

Canuto, V. \& Mazzitelli, I. 1992, ApJ, 389, 724 
Carretta, E., Gratton, R., Clementini, G., \& Fusi Pecci, F. 1999, ApJ, submitted [astro-ph/9902086]

Castelli, F. 1999a, private communication

Castelli, F. 1999b, A\&A, 346, 564

Chaboyer, B., Demarque, P., Kernan, P. J., \& Krauss, L. M. 1998, ApJ, 494, 96

Chadid, M. \& Gillet, D. 1998, A\&A, 335, 255

Chieffi, A., Limongi, M., \& Straniero, O. 1998, private communication

Cole, A. A. 1998, ApJ, 500, L137

Dorman, B., Rood, R. T., \& O'Connell, R. 1993, ApJ, 419, 596

Feast, M. W. \& Catchpole, R. M. 1997, MNRAS, 286, L1

Fernley, J. 1994, A\&A, 284, L16

Fernley, J., Carney, B. W., Skillen, I., Cacciari, C., \& Janes, K. 1998, MNRAS, 293, L61

Ferraro, F. R., Messineo, M., Fusi Pecci, F, et al. 1999, AJ, in press [astroph/9906248]

Fuhrmann, K., Axer, M., \& Gehren, T. 1993, A\&A, 271, 451

Gardiner, R. B., Kupka, F., \& Smalley, B. 1999, A\&A, 347, 876

Gautschy, A. 1987, Vistas in Astron., 30, 197

Gould, A. \& Popowski, P. 1998, ApJ, 508, 844

Gratton, R. 1998, MNRAS, 296, 739

Gratton, R., Fusi Pecci, F., Carretta, E., et al. 1997, ApJ, 491, 749

Grundahl, F., VandenBerg, D. A., \& Andersen, M. I. 1998, ApJ, 500, L179

Guinan, E. F., Fitzpatrick, E. L., Dewarf, L. E., et al. 1998, ApJ, 509, L21

Jones, R. V., Carney, B. W., Latham, D. W., \& Kurucz, R. L. 1987, ApJ, 312, 254

Jones, R. V., Carney, B. W., Storm, J., \& Latham, D. W. 1992, ApJ, 386, 646

Kovács, G. \& Walker, A. R. 1998, ApJ, 512, 271

Kurucz, R. L. 1979, ApJS, 40, 1

Kurucz, R. L. 1995, available at http://cfaku5.harvard.edu

Lee, M. G., Freedman, W. L., \& Madore, B. F. 1993, ApJ, 417, 553

Liu, T. \& Janes, K. A. 1990, ApJ, 354, 273

Martin, J. C. \& Morrison, H. L. 1998, AJ, 116, 1724

Montegriffo, P., Ferraro, F. R., Origlia, L., \& Fusi Pecci, F. 1998, MNRAS, 297, 872

Oke, J. B., Giver, L. P., \& Searle, L. 1962, ApJ, 136, 393

Panagia, N. 1998, Mem. S. A. It., 69, 225

Pont, E., Mayor, M., Turon, C., \& VandenBerg, D. A. 1998, A\&A, 329, 87

Reid, I. N. 1997, AJ, 114, 161

Sandage, A. 1993, AJ, 106, 703

Skillen, I., Fernley, J., Stobie, R. S., \& Jameson, R.F. 1993, MNRAS, 265, 301

Sweigart, A. W. 1997, ApJ, 474, L23

Udalski, A., Pietrzynski, G., Wozniak, P., et al. 1998, ApJ, 509, L25 